Article

\title{
The Languages of Neurons: An Analysis of Coding Mechanisms by Which Neurons Communicate, Learn and Store Information
}

\author{
Morris H. Baslow \\ Nathan S. Kline Institute for Psychiatric Research, Center for Neurochemistry, 140 Old Orangeburg \\ Road, Orangeburg, NY 10962, USA; E-Mail: Baslow@nki.rfmh.org; \\ Tel.: +1-845-398-5471; Fax: +1-845-398-5472
}

Received: 17 August 2009 / Accepted: 30 October 2009 / Published: 4 November 2009

\begin{abstract}
In this paper evidence is provided that individual neurons possess language, and that the basic unit for communication consists of two neurons and their entire field of interacting dendritic and synaptic connections. While information processing in the brain is highly complex, each neuron uses a simple mechanism for transmitting information. This is in the form of temporal electrophysiological action potentials or spikes (S) operating on a millisecond timescale that, along with pauses $(\mathrm{P})$ between spikes constitute a two letter "alphabet" that generates meaningful frequency-encoded signals or neuronal S/P "words" in a primary language. However, when a word from an afferent neuron enters the dendritic-synaptic-dendritic field between two neurons, it is translated into a new frequency-encoded word with the same meaning, but in a different spike-pause language, that is delivered to and understood by the efferent neuron. It is suggested that this unidirectional inter-neuronal language-based word translation step is of utmost importance to brain function in that it allows for variations in meaning to occur. Thus, structural or biochemical changes in dendrites or synapses can produce novel words in the second language that have changed meanings, allowing for a specific signaling experience, either external or internal, to modify the meaning of an original word (learning), and store the learned information of that experience (memory) in the form of an altered dendritic-synaptic-dendritic field.
\end{abstract}

Keywords: biosemiotics; codes; cognition; language; learning; memory; neurons; semiosis 


\section{Introduction}

Semiosis is broadly defined as any form of activity, conduct or processes that involve signs including the production of meaning, and semiotics is broadly defined as a study of these processes and their significance in communication. A semiotic system has also been more narrowly defined [1,2] as consisting of signs, meanings, a code and a codemaker and it has been proposed that this code model is a first step toward a scientific understanding of biosemiotics, or biology interpreted as a sign system study, a classification based on the general assertion that all living organisms are semiotic systems. Further, that biosemiotics as a recognizable scientific discipline requires proof that the individual cell is a semiotic system [1]. Based on the more narrow definition, the codemaker is described as the genetic code which determines the nature of proteins synthesized by cells, and that signs and meanings are codemaker-dependent entities. In this article, both the broader and more narrow definitions of semiotics at the cellular level are considered, and an attempt is made to bring together current research in the natural sciences on how cells communicate, learn and store information.

In multi-cellular animals, neurons are cells specialized for rapid communication of meaningful information, and their interactions on the cellular level form the basis for semiosis at all higher levels of complexity in all animal forms of life. In this paper evidence is provided that individual neurons possess language (production of meaningful signs), based on codemaker generated "sensors" and that the basic unit for communication (interpretation of signs) consists of two neurons and their field of interacting dendritic and synaptic connections. In order to better understand biosemiotic interactions between neurons there are several premises which form the basis for such an analysis. These are:

1. Encoded messages sent by afferent neurons are meaningful.

2. Messages received by efferent neurons can be interpreted.

3. Messages received by efferent neurons can be acted upon.

4. There is a feedback system between neurons to ascertain successful communication.

5. Meaningful messages can be changed to new meaningful messages that reflect useful changes induced by either external or internal experiences.

6. Communication is an energy consuming process.

While the structure of neural codes in complex systems remains to be resolved $[3,4]$, in the following sections, coding methods used by individual neurons to communicate are explored, and possible mechanisms whereby messages can be altered by experience (learning) and the learned information of that experience (memory) can be stored over long periods of time for use in higher cognitive functioning.

\section{Neuronal Languages}

\subsection{Energy requirements for communication}

The basic function of neurons is to communicate, and they do this by generating coherent intracellular electrophysiological signals, in the form of action potentials (AP's) or "spikes", at various encoded frequencies [5,6]. These signals are then translated into a variety of frequency-encoded neurochemical signals transmitted to other neurons at synapses, and subsequently interpreted at some 
level in the central nervous system (CNS) neural network [7,8]. Since formation of spikes is an energy expensive process, calculated as using $2.2 \times 10^{9}$ adenosine triphosphate (ATP) molecules per spike [9], it has been proposed that AP's are generated only when required for specific tasks and that neurons are electro-physiologically inactive most of the time

\subsection{Neuronal language formats}

While information processing in the brain is highly complex, each neuron uses a simple code mechanism for transmitting information. This is in the form of temporal electrophysiological action potentials or spikes $(\mathrm{S})$ of about a 1 millisecond $(\mathrm{ms})$ duration that, along with pauses $(\mathrm{P})$ between spikes constitute a two letter "alphabet" that generates meaningful frequency-encoded signals or neuronal S/P "words" in a primary language. The term "word" for neuronal activity in spikes/s has previously been used to describe the electrophysiological activity of the visual interneuron of the blowfly where each AP was considered a "short" word and trains of AP's considered "longer" words [10]. In this article a neuronal word is defined as a single AP together with the pause before the next AP (the "short" word) in a ms timeframe. The "longer" word is defined as a "phrase" consisting of a repeated group of two or more short words preceded by, and followed by pauses, and a sentence as a temporal grouping of such words containing a subject, and a predicate that expresses what is stated about the subject. All neuron codes, whether continuous trains of spikes that are characteristic of information transmitted at low frequencies, or bursts of spikes that are characteristic of information transmitted at high frequencies, are made up of interactions between spikes and pauses.

There are two theories regarding how these spikes carry encoded information [7]. One is the "spike rate code" which suggests that information is carried in the average rate at which the neuron fires, and that the timing of each spike is random. The other is the "spike timing code" that suggests that specific information is carried not only in the average rate at which a neuron fires, but also in the precise timings between each spike. While it is difficult to investigate how information might be encoded within the intact CNS [3], a study of how some receptors sense and transmit information is informative. In a study of the isolated salt receptor of the blowfly [11], it was observed that information about $\mathrm{NaCl}$ molarity was transmitted using a frequency code in the range of 2-120 Hertz (Hz), evident as a linear association between the mean receptor frequency response and the logarithm of molarity (spike rate code). From these results it is also possible to derive the $\mathrm{S} / \mathrm{P}$ encoded words in a millisecond timeframe (spike timing code) that is associated with each $\mathrm{NaCl}$ molar concentration experienced by the receptor (Table 1).

Coding mechanisms of the salt, as well as other taste receptors of the fruit fly are similar to that of the blowfly, and have been reviewed [13]. Similar responses have been observed in hamster fungiform taste receptor cells exposed to acid stimulation, where the frequency of spikes varies directly with increasing acidity between $\mathrm{pH} 2.5$ to 5.5 [14]. In this case, the frequency was almost linear, with a mean frequency response of $3.5 \mathrm{~Hz}\left(\mathrm{~S}_{1} \mathrm{P}_{286}\right)$ at $\mathrm{pH} 2.5$, and a frequency of $0.25 \mathrm{~Hz}\left(\mathrm{~S}_{1} \mathrm{P}_{4000}\right)$ at $\mathrm{pH} 5.0$. In a study of the responses of receptor B-cells of the antennal taste sensilla of the ground beetle to $\mathrm{NaCl}$ and several other salts, the rate of spiking was observed to be increased directly with $\mathrm{NaCl}$ concentrations of $0.001,0.01,0.10$ and $1.00 \mathrm{M}$ resulting in spiking codes of approximately $\mathrm{S}_{1} \mathrm{P}_{1000} ; \mathrm{S}_{1} \mathrm{P}_{71} ; \mathrm{S}_{1} \mathrm{P}_{29}$ and $\mathrm{S}_{1} \mathrm{P}_{23}$ respectively [15]. Importantly, these cells were also able to distinguish between the different salts tested including $\mathrm{NaCl}, \mathrm{KCl}$, sodium acetate, $\mathrm{CaCl}_{2}$ and $\mathrm{MgCl}_{2}$ evident by their production of different 
spiking codes for each salt. Thus, use of S/P language in this case was clearly quite rich in the meanings that could be transmitted.

Table 1. Response of the isolated salt receptor of the blowfly to the molar concentration of $\mathrm{NaCl}$ at $25.5{ }^{\circ} \mathrm{C}$ and $80 \%$ relative humidity ${ }^{\mathrm{a}}$.

\begin{tabular}{llll}
\hline $\begin{array}{l}\text { NaCl } \\
\underline{(\mathbf{M})}\end{array}$ & $\begin{array}{l}\text { Signal }[\mathbf{H z}] \\
\text { (spikes/s) }\end{array}$ & $\begin{array}{l}\text { Relative } \\
\text { refractiveness } \\
\text { (ms between spikes) }\end{array}$ & $\begin{array}{l}\text { S/P encoded word } \\
\text { (ms time frame) }\end{array}$ \\
\hline 0.10 & 2 & 499 & $\mathrm{~S}_{1} \mathrm{P}_{499}$ \\
0.20 & 22 & 45 & $\mathrm{~S}_{1} \mathrm{P}_{45}$ \\
0.25 & 36 & 27 & $\mathrm{~S}_{1} \mathrm{P}_{27}$ \\
0.50 & 52 & 18 & $\mathrm{~S}_{1} \mathrm{P}_{18}$ \\
1.00 & 80 & 12 & $\mathrm{~S}_{1} \mathrm{P}_{12}$ \\
2.00 & 104 & 9 & $\mathrm{~S}_{1} \mathrm{P}_{9}$ \\
3.00 & 120 & 7 & $\mathrm{~S}_{1} \mathrm{P}_{7}$ \\
\hline \multicolumn{5}{c}{} & & \\
& ${ }^{\mathrm{b}}$ Adapted from $[11]$ & &
\end{tabular}

Single glucosensing neurons in the hypothalamus of the brain can sense the concentration of glucose (Glc) in their environment and respond with changes in spiking frequency [16-18]. These neurons have been observed in both satiety and feeding centers of cat and dog brain, and their responses in these centers mirror one another [16]. In the satiety center of cats, $30 \mathrm{~min}$ after intra-venous Glc administration, the mean spike frequency rose from $0.1 \mathrm{~Hz}\left(\mathrm{~S}_{1} \mathrm{P}_{10000}\right)$ to $0.5 \mathrm{~Hz}\left(\mathrm{~S}_{1} \mathrm{P}_{2000}\right)$. In the feeding center under these same conditions, the mean spike frequency fell from $0.4 \mathrm{~Hz}\left(\mathrm{~S}_{1} \mathrm{P}_{2500}\right)$ to $0.1 \mathrm{~Hz}$ $\left(\mathrm{S}_{1} \mathrm{P}_{10000}\right)$. The function of glucosensing neurons clearly satisfies both the biosemiotic code requirements and the premises outlined for cellular communication presented in section 1. With regard to premise three, the ability to act on the content of the message, glucosensing neurons and their efferent targets have the ability to signal the vascular system to increase or decrease the supply of Glc. They do this by releasing a variety of relatively non-excitotoxic neurotransmitters into ECF when stimulated, that in turn activate their glial chaperone cells [19]. When astroglial cells are activated, they release second chemical messengers to the vascular endothelial cells of capillary beds that results in a timely increase in focal blood flow (hyperemia) and a concomitant increase in available Glc [20].

If we use a dictionary definition and define language as the "transmission of ideas between any living creatures by any means", it is apparent that individual neurons use language to communicate with other neurons. The sending language is then projected in the form of a two letter alphabet consisting of spikes and pauses between spikes that form specific meaningful reproducible "words" that can be used to transmit information obtained from a variety of external and internal sensors. The kind of information transmitted is sensor-dependent, but specific S/P languages can be used to identify and transmit both qualitative and quantitative information provided by the sensors. Within the limits of these studies, the investigators, as well as the post-synaptic cells, can not only "read" the encoded language, but we can 
also interpolate the data sets and "write" additional meaningful $\mathrm{S} / \mathrm{P}$ words. This coding mechanism can also accommodate both continuous and "bursting" signaling within a syntax that includes short temporal phrases and longer sentences composed and communicated within a millisecond timeframe. There is a also a grammar in that neurons generally use a simple imperative made up of a single word in their communications, and sentences are generated in real-time to describe changing conditions.

Of interest, the S/P code can also be written in terms of electronically generated musical "notes" based on their frequency equivalents, and then applying established musical conventions for notes, including temporal note lengths, and rests $(\mathrm{R})$ between notes. In this analogy, phrases and sentences can be represented in terms of sequences of specific notes with different notes representing an informational melody. In Table 2, the frequency data for the isolated blowfly salt receptor is presented in $\mathrm{Hz}, \mathrm{S} / \mathrm{P}$ words and in their musical notations.

Table 2. Response of the isolated salt receptor of the blowfly to the molar concentration of $\mathrm{NaCl}$ at $25.5^{\circ} \mathrm{C}$ in $\mathrm{Hz}, \mathrm{S} / \mathrm{P}$ words and in frequency-equivalent musical notations.

\begin{tabular}{ccccc}
\hline $\begin{array}{c}\text { NaCl } \\
\underline{(\mathbf{M})}\end{array}$ & $\begin{array}{c}\text { Signal }[\mathrm{Hz}] \\
\text { (spikes/s) }\end{array}$ & $\begin{array}{c}\text { S/P encoded word } \\
\text { (ms time frame) }\end{array}$ & \multicolumn{2}{c}{$\begin{array}{c}\text { Musical notations } \\
\text { (equal-tempered scale) }\end{array}$} \\
\hline 0.20 & 22 & $\mathrm{~S}_{1} \mathrm{P}_{45}$ & 21.83 & $\underline{\text { Note }}$ \\
0.25 & 36 & $\mathrm{~S}_{1} \mathrm{P}_{27}$ & 36.71 & $\mathrm{~F}_{0}$ \\
0.50 & 52 & $\mathrm{~S}_{1} \mathrm{P}_{18}$ & 51.93 & $\mathrm{G}_{1}^{\#}$ or A $_{1}^{\mathrm{b}}$ \\
1.00 & 80 & $\mathrm{~S}_{1} \mathrm{P}_{12}$ & 82.41 & $\mathrm{E}_{2}$ \\
2.00 & 104 & $\mathrm{~S}_{1} \mathrm{P}_{9}$ & 103.83 & $\mathrm{G}_{2}^{\#}$ or $\mathrm{A}_{2}^{\mathrm{b}}$ \\
3.00 & 120 & $\mathrm{~S}_{1} \mathrm{P}_{7}$ & 123.47 & $\mathrm{~B}_{2}$ \\
\hline
\end{tabular}

${ }^{\mathrm{a}}$ These notes are for music frequencies in an equal-tempered scale based on $\mathrm{A}_{4}$ being equal to $440 \mathrm{~Hz}$. The musical notations above closely approximate the $\mathrm{S} / \mathrm{P}$ encoded words in terms of transmission frequencies. The musical notation for $587.33 \mathrm{~Hz}$, a frequency value at the upper range of rates of neuronal signaling, is $\mathrm{D}_{5}$.

Based on this analogy, it would appear that neurons may in fact not only communicate with one another using meaningful words in a variety of neuronal languages, but may also transmit information in the form of "singing" silently to one another. Neurophysiologists are routinely able to detect, visualize and listen to neuronal electromagnetically-generated communications that are produced by individual neurons.

\subsection{Dictionary of neuronal words and the universal nature of their translatability}

The dictionary of neuronal S/P words in an ms timeframe is relatively short, being limited at its upper level by the absolute and relative neuronal refractory periods and at its lower level by physiological requirements such as maintenance of the human heart rate at 68 beats/min $(1.13 \mathrm{~Hz})$. With the average spike including an absolute refractory period of $1 \mathrm{~ms}$ duration followed by a relative refractory period of about $2 \mathrm{~ms}$, the highest possible frequency would be $\mathrm{S}_{1} \mathrm{P}_{2}$ or $333 \mathrm{~Hz}$. If the system were driven by a strong enough input eliminating the relative refractory period altogether, the highest possible frequency might be $S_{1} P_{0}$ or $1,000 \mathrm{~Hz}$. Since the brain's primary form of communication, both internally and with the outside world, is in the form of electrophysiological messages, it follows that neuronal words are 
highly representational in nature. For example, audible sound in many animals may range from $5,000-10,000 \mathrm{~Hz}$ and in some animals may reach up to $20,000-30,000 \mathrm{~Hz}$, and visible light is in the range of $10^{14} \mathrm{~Hz}$, both of which radiations must be converted into meaningful words of less than $1,000 \mathrm{~Hz}$ for transmission to and within the CNS. Although the upper range of frequency-encoded messages is limited, the richness of possible responses is almost unlimited in that the temporal length of individual spikes, and of pauses can vary within a sub-millisecond time period. In this article, the S/P codes presented are averages that have been rounded to the nearest ms. However, information may be embedded in fractions of a ms, as well as in rhythmic oscillatory functions that occur during temporal $\mathrm{S} / \mathrm{P}$ word sequences. For example, at a spike length of $0.5 \mathrm{~ms}$ at $120 \mathrm{~Hz}$, $\mathrm{P}=[1,000 \mathrm{~ms}-(120 \times 0.5)] / 120$ and the encoded word would be one spike followed by a $7.83 \mathrm{~ms}$ pause $\left(\mathrm{S}_{0.5} \mathrm{P}_{7.83}\right)$. At a $\mathrm{S}_{\mathrm{L}}$ of $1.0 \mathrm{~ms}$, the encoded word would be $\mathrm{S}_{1} \mathrm{P}_{7.33}$, and at a $\mathrm{S}_{\mathrm{L}}$ of $1.5 \mathrm{~ms}$, the encoded word would be $\mathrm{S}_{1.5} \mathrm{P}_{6.83}$. Using this same illustration, rhythmic oscillations around the $120 \mathrm{~Hz}$ frequency would further modify the $\mathrm{S} / \mathrm{P}$ encoded words and thus introduce additional information-carrying potential. If every fifth $\mathrm{AP}$ at a $\mathrm{S}_{\mathrm{L}}$ of $1.0 \mathrm{~ms}$ was at $100 \mathrm{~Hz}$, the fifth word in the phrase would be $S_{1} P_{9.00}$ instead of $S_{1} P_{7.33}$. Since AP generation is an energy requiring process, bioenergetic factors would appear to favor the lowest frequencies for the shortest periods of time for transmission of information.

Based on the three frequency-encoded language formats presented in Table 2, a hypothetical bursting series consisting of two phrases of $100 \mathrm{~ms}$ of $104 \mathrm{~Hz}\left(\mathrm{~S}_{\mathrm{L}}\right.$ of $\left.1.0 \mathrm{~ms}\right)$ word bursts separated by a $100 \mathrm{~ms}$ pause in $\mathrm{Hz}, \mathrm{S} / \mathrm{P}$ and music notations would be:

$(\mathrm{Hz})$

$\left[(104 \mathrm{~Hz})_{100},(0 \mathrm{~Hz})_{100},(104 \mathrm{~Hz})_{100}\right]$
$(\mathrm{S} / \mathrm{P})$

$\left[\left(\mathrm{S}_{1} \mathrm{P}_{9}\right)_{10},(\mathrm{P})_{100},\left(\mathrm{~S}_{1} \mathrm{P}_{9}\right)_{10}\right] \quad\left[\left(\mathrm{G}_{2}^{\#}\right)_{100},(\mathrm{R})_{100},\left(\mathrm{G}_{2}^{\#}\right)_{100}\right]$
(Music)

with the two phrase sentence punctuated by a pause at the end of the bursting event. This frequency-encoded sentence sent to the insect's CNS in "Flyspeak" could also reasonably be translated into the English language as:

"A salt receptor (the subject) on the tip of my labellar sensillum has been exposed to a $2 \mathrm{M}$ concentration of $\mathrm{NaCl}$ for a period of $300 \mathrm{~ms}$ ( the predicate)!"

The universal nature of neuronal S/P languages is clear in that they can be written in many different frequency-encoded formats, and can be translated into any number of spoken or symbolic human languages, all with the same meaning. Neurons are also highly specialized communicating cells that use both electrophysiology, and what appears to be a more primitive chemical communication mechanism between neurons at chemical synapses. Thus, it follows that inter-cellular chemical communication that is used by a variety of modern cells as well as neurons is an ancient trait, and that all cells are probably biosemiotic systems.

\subsection{Communication with neurons using electronically-generated frequency-encoded messages}

Of importance, it has recently been demonstrated that neuronal S/P frequency-encoded words in response to selected stimulants carry messages to the CNS that result in specific behavioral responses. 
In rats, the $\mathrm{S} / \mathrm{P}$ word response of taste cells to quinine at $0.01 \mathrm{mM}\left(\mathrm{S}_{1} \mathrm{P}_{21}\right)$ was associated with an avoidance behavior to the licking of water containing this bitter substance. When the S/P cellular quinine word response was mimicked by patterned electrical pulse trains, the electronically generated pulse trains sent to the CNS were found to elicit the same behavioral avoidance response as the cell-generated S/P word trains, underscoring the functional significance of temporal S/P coding [21]. These results also suggest that knowledge of specific neuronal codes might be a useful adjunct in treatment of certain human neuropathies, perhaps similar to the methodologies currently used in cardiac pacemaker technology where an electronic message at $1.13 \mathrm{~Hz}$ translates into 68 heartbeats/min.

\section{Inter-Neuronal Transmission of Information}

The physiological function of neurons is communication, and neurons carry out this function by transmitting frequency-encoded information to other neurons in the form of simple or complex trains of impulses. Briefly stated, each afferent neuron propagates membrane bioelectric signals that are passed along their neuronal soma, axon extension, and then their dendritc arborizations in the form of rapid de-polarizations and re-polarizations in a frequency range generally between $0.1-600 \mathrm{~Hz}$. The afferent neuron then transmits the frequency-encoded signals to the efferent neuron across synaptic space by converting the bioelectric signals into chemical signals using a variety of neurotransmitters released from pre-synaptic vesicles into the synaptic space. These neurotransmitter molecules then diffuse about 20-30 nm to post-synaptic membranes of the receiving neuron [22]. Synapses constitute a highly confined extracellular fluid (ECF) compartment, being only about 50-100 water molecules wide, and neurotransmitters, upon docking with post-synaptic receptors, must be recycled in a timely manner in order to reactivate the receptors for additional signaling. Although in some cases neurons can form electrical synapses with other neurons via gap junctions [23], chemical synapses are the predominant form used for inter-neuronal communication. Where electrical synapses between neurons are utilized, their function appears to be to induce synchrony in the activity of a number of afferent neurons prior to using chemical synapses for transmission of information to efferent neurons.

The post-synaptic neuron receives the original frequency-encoded message in the form of temporal bursts of chemical transmitter arriving at specific post-synaptic surface receptors which then generate bioelectric signals carried along the receiving neuron dendritic arborization pathways to a common somatic axonal terminus. At this terminus, the S/P messages from the various dendrites may reform into a new S/P word, which is then passed onto the efferent neuron soma and its axon extension for further propagation and/or response to the interpreted message. Clearly, in this sequence the synapses are a vital component in the transmission of meaningful information since they are where encoded bioelectric signals are translated into encoded chemical signals and back into encoded bioelectric signals once again. As such, synapses are particularly important sites in information transfer. However, it has been proposed that it is not synapses alone, but the entire dendritic-synaptic-dendritic field between neurons that plays an important role in inter-neuronal communication [24-28]. If only one dendritic pathway and one synapse were involved between two neurons, the original message might be received by the efferent neuron in its original spike-pause encoded word form, but even in such a case, since the dendritic-synaptic interface is a dynamic and continuously regenerating system, it could also be 
modulated by changes in single pathway elements. The basic structure of a single dendritic-synaptic-dendritic connection between two neurons is illustrated in Figure 1.

Figure 1. Structural and biochemical components associated with a single dendriticsynaptic-dendritic pathway between neurons.

\section{Sending neuron}
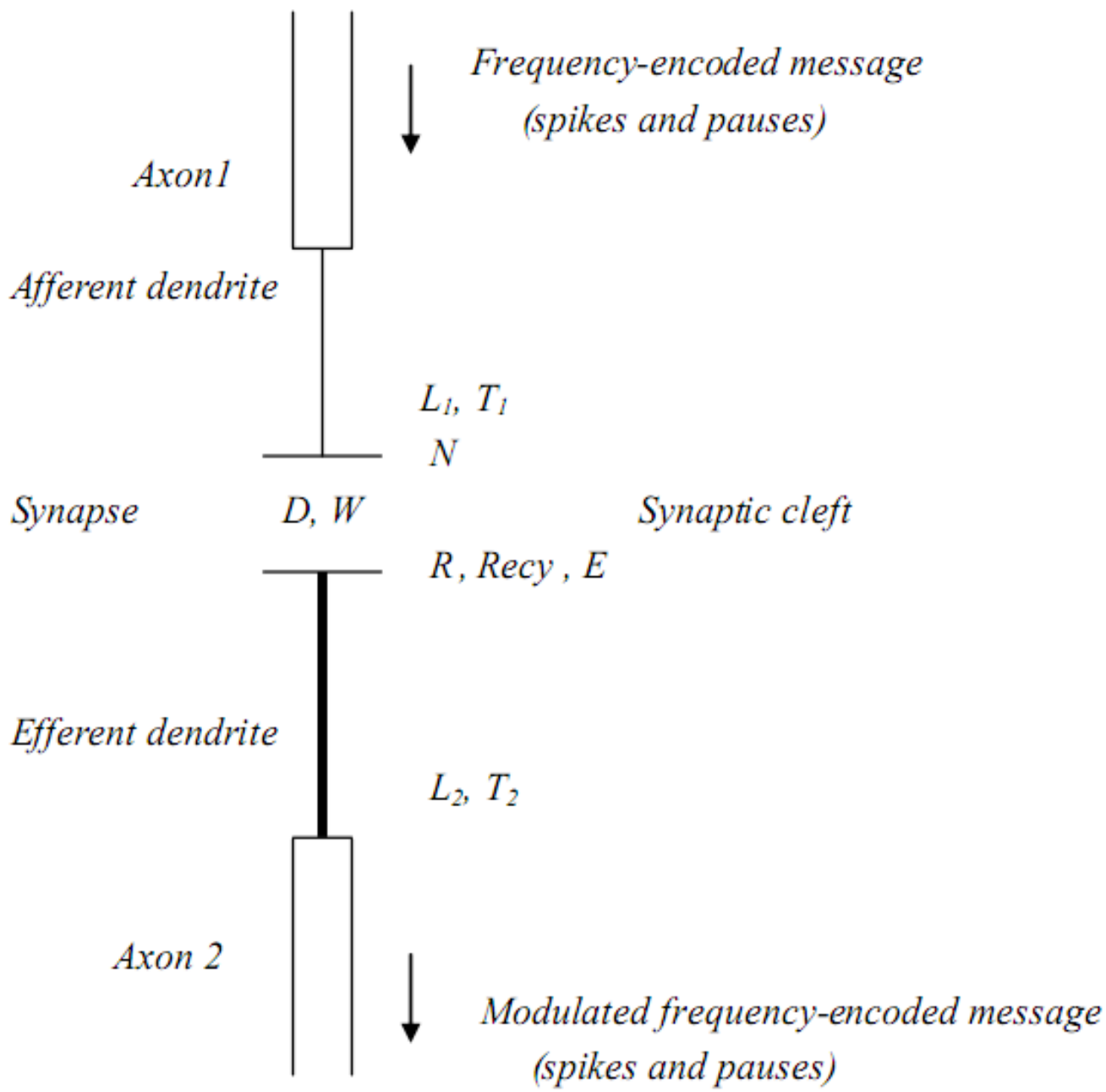

Receiving neuron

In this illustration, the length $(L)$ of each dendrite, thickness $(T)$ of each dendrite, synaptic cleft distance $(D)$, and synaptic cleft width $(W)$ are all seen to be potential structural sites of signal modulation. At the synapse itself there are additional chemical and biochemical sites for modulation. These include the rates and types of neurotransmitters $(N)$ released, the number and types of receptors $(R)$ available, energy $(E)$ availability and the enzymatic and/or transporting mechanisms available for neurotransmitter recycling (Recy). These latter components can delay, and/or alter the frequency of transmitted messages in that they govern the rate of clearance of neurotransmitters, and therefore affect the synaptic re-polarization time [29]. If the synaptic re-polarization time is greater than the message frequency, the frequency will be truncated and affect the signal that reaches the receiving neuron $[8,30]$. 
In Figure 2 the basic structure of a chemical synapse is illustrated, as well as how Fick's law interacts with how a transmitter diffuses across the synaptic cleft. Fick's law; $\mathbf{J}=\mathbf{D A}\left[\mathbf{C}_{\mathbf{1}}-\mathbf{C}_{\mathbf{2}} / \boldsymbol{\Delta} \boldsymbol{x}\right]$ incorporates the rate of diffusion (D) of the hydrated transmitter, the width of the synaptic space $(\Delta x)$, the cross-sectional area (A) of the space, and the concentrations of transmitter at the pre-synaptic membrane $\left(\mathrm{C}_{1}\right)$ and post-synaptic membrane $\left(\mathrm{C}_{2}\right)$, with $\left[\mathrm{C}_{1}-\mathrm{C}_{2} / \Delta x\right]$ being the transmitter concentration gradient. $(\mathrm{J})$ is the rate of mass movement of the transmitter whose time of arrival at the post-synaptic membrane is a function of $(\Delta x)$. Surrounding the synapse are astrocyte endfeet rich in aquaporin- 4 (AQP4) water channels. If $(\Delta x)$ changes, the timing of information-embedded signal trains arriving at the post-synaptic membrane will be altered and normal cognition may be impaired. Astrocyte AQP4 water channels play an important role in this timing process in that they effectively regulate the water content of the synaptic cleft, thus maintaining its structural stability and the dimension of $(\Delta x)$. In this simple representation of a synapse, only a few of the many structural and biochemical variables have been included.

Figure 2. Synaptic structure and Fick's law, as it applies to synaptic function.

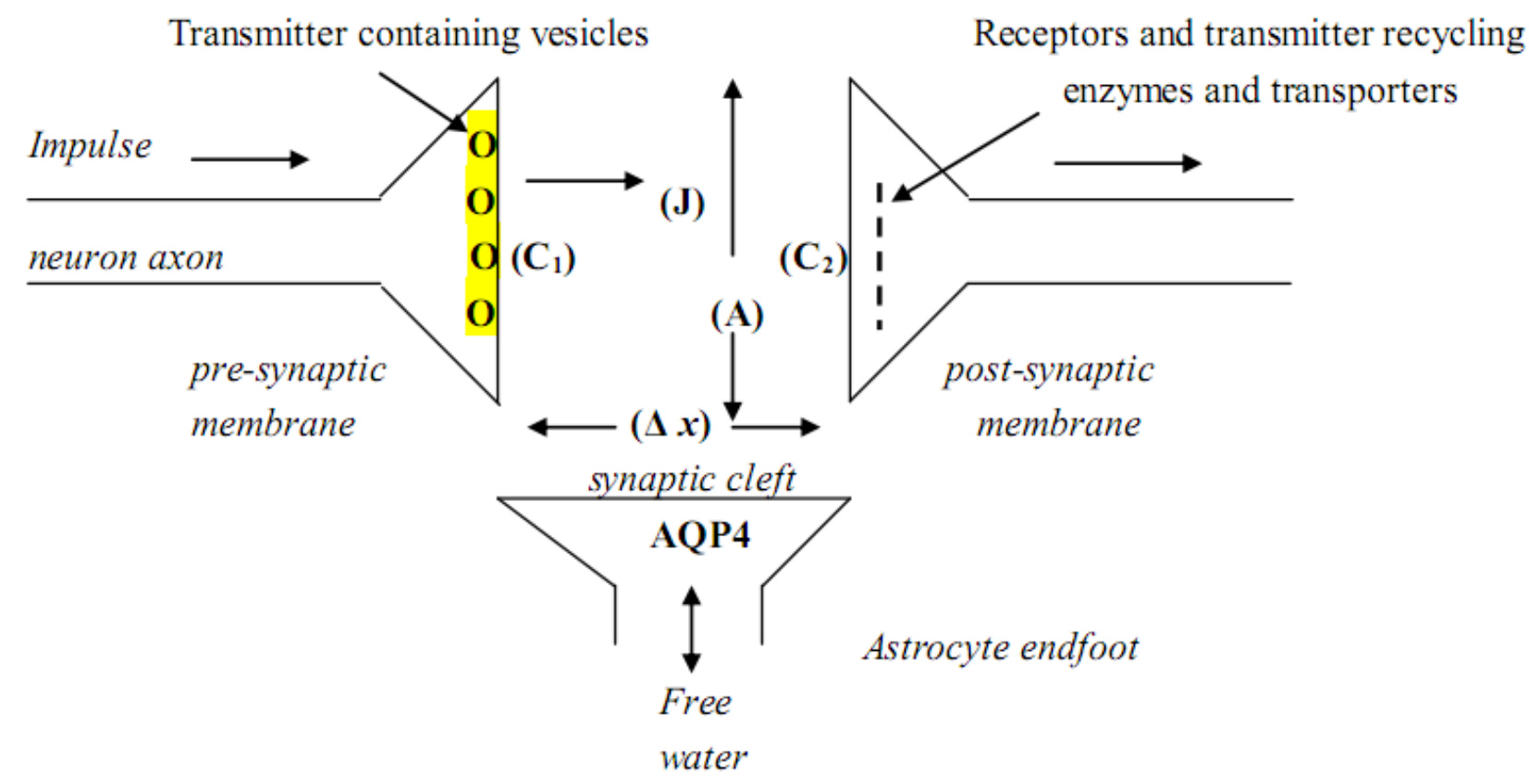

Multiple arborizations and connectivity between two neurons is the typical arrangement, and as such can play an important role in cognition. Moreover, in the CNS, the situation is still more complex in that signals sent by an afferent neuron may be sent to many other neurons, and signals received by an efferent neuron may have multiple neuronal sources. In addition, glial chaperone cells can also interact with elements of synaptic transmission [31,32]. Nonetheless, brain function while extremely complex is not chaotic and the basic premises outlined in section 1 must still apply if normal cognitive functioning is to be achieved.

If more than one dendrite and synapse are involved between two neurons, it is highly unlikely that the original message will be received by the efferent neuron in its original spike-pause encoded form. Thus, modulation of the message is probably a major component of all inter-neuronal communications. 
In Figure 3, an inter-neuronal association with multiple dendritic-synaptic-dendritic connections is illustrated.

Figure 3. The basic unit of cognitive function; two neurons and their dendritic arbors and synapses.

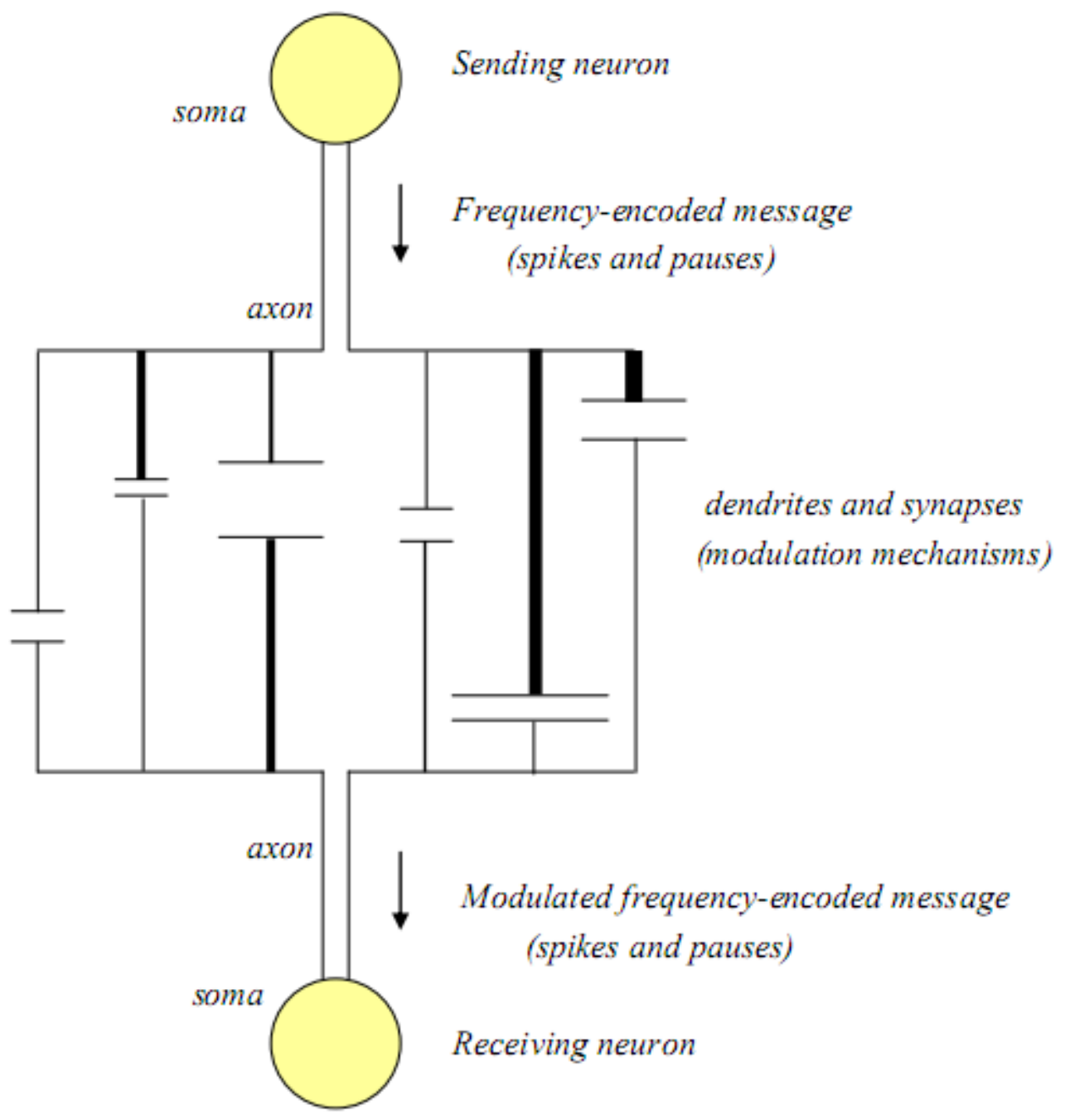

In this illustration, the number of dendritic-synaptic pathways has been expanded, and each pathway is pictured as being unique, having different lengths, different dendritic thicknesses, as well as different synaptic widths and synaptic cleft distances. In this model, all of the rate-limiting steps associated with each single dendritic-synaptic-dendritic pathway still operate, and as a result they interact to affect the signals that reach the receiving neuron, where the incoming signals are reassembled into a new spike-pause code which may be delayed and/or frequency modulated compared to the original message.

\section{Importance of the Inter-Neuronal Language Translation Step to Cognitive Function}

\subsection{Original neuronal S/P words are modulated upon passage through a dendritic-synaptic field}

There is evidence that when a meaningful spike-pause word from an afferent neuron enters a multiple dendritic-synaptic-dendritic field between two neurons, the original S/P word is modulated [7,8,33,34] 
and translated into a new equivalent meaningful frequency-encoded word in a different spike-pause language. Based on the variables in a given dendritic-synaptic-dendritic field between any two neurons, it is highly improbable that any original afferent neuron encoded S/P signal when received by an efferent neuron will ever be in its original state. Thus, it is postulated that this $\mathrm{S} / \mathrm{P}$ word translation step is a normal function in all neuronal communications. It is also important to consider that while the original encoded message may have meaning in itself, that the meaning is not a function of its interpretation. Thus, under some pathological or drug-induced conditions, receiving neurons may not be able to decipher and act on meaningful encoded messages that are transmitted to it.

\subsection{A potential role for inter-neuronal S/P language modulation in learning and memory}

It is proposed that the observed unidirectional inter-neuronal language-based word translation step is of utmost importance to brain function in that it allows for variations in meaning to occur. Thus, any structural or biochemical changes in the dendritic-synaptic-dendritic field can produce novel words in the second language that have changed meanings, allowing for a specific signaling experience to modify the meaning of an original word (learning), and store the learned information of that experience (memory) in the form of an altered dendritic-synaptic-dendritic field. This conforms with the notion that neurons use a natural language for their inter-cellular communications, a concept that is further supported by evidence of plasticity associated with enriched environmental stimuli in mammals that are known to result in altered electrophysiological, structural and biochemical elements of dendritic-synaptic-dendritic field connections between neurons [35-37]. Since the mechanism that brings about such changes is a function of the level of use of specific neuronal pathways that in turn induce genotype-linked phenotypic modifications, it follows that any source of input, external or internal can initiate these changes. This inter-neuronal modulation step is of importance in that it allows brain activities of all kinds and at all levels, whether from external sensors such as eyes and ears, or internally generated as "thought processes" to result in changes in neuronal words in response to increased or decreased use of particular neuronal pathways. The concept of the diversity of dendritic excitability that arise from variations in dendrite morphology and channel distribution between neurons as illustrated in Figure 3, and the potential role of such diversity in information processing and memory storage in neuronal networks have been considered in detail [24,25,27,28] and extensively reviewed [26].

\subsection{Mechanisms of neuronal plasticity that can affect cognitive function at the two-cell level}

Neuronal languages when transmitted to a recipient neuron constitute a biosemiotic mechanism at the cellular level that is made up of words in S/P encoded frequencies. These words are clearly rate-sensitive, and several of the specific sites and elements in the inter-neuronal field that might be altered (plasticity) by changes in environmental stimuli, internal brain activity, pathological conditions or by use of therapeutic drugs [38] are listed in Table 3. 
Table 3. Potential sites of plasticity in inter-neuronal signaling.

Electrophysiological components

Sensor initiated frequency-encoded messages

Temporal length of the communications

Inhibitory feedback circuits associated with pre-synaptic receptors

Coincidence of arrival of excitatory post-synaptic potentials

Number of available dendritic pathways that are activated

Structural components

Number and types of environmental sensors

Length of afferent-efferent dendritic pathways

Width of synaptic clefts

Distance between pre and post-synaptic membranes

Number of afferent-efferent dendritic pathways

Thickness of afferent or efferent dendrites

Position of pre-synaptic release of neurotransmitters

Makeup of post-synaptic cleft protein densities

Number and types of post-synaptic membrane receptors

Number and types of post-synaptic membrane transporters

Biochemical components

Types of neurotransmitters used for signaling

Number of neurotransmitter molecules per synaptic vesicle

Presence of multiple neurotransmitters in a single vesicle

Quantities of neurotransmitters released into the synaptic cleft

Specific enzyme activities associated with transmitter recycling mechanisms

\subsection{Reflections on the nature and site of learning and memory engrams}

In this article, evidence has been provided in support of the biosemiotic notion that neurons communicate with each other using language that has an alphabet, specific words and structured sentences. From this evidence, one can also reflect on the nature of learning and of acquired memories. Since formation of neuronal words is a energy expensive process [9], it has been proposed that AP's are generated only when required for specific tasks. Bioenergetic constraints also indicate that available ATP can only support an average spike rate in human cortical neurons of $0.16 \mathrm{~Hz}$ [9] such that neurons are in the pause phase $99.98 \%$ of the time. Therefore it is logical that neuronal S/P words do not exist as such in the CNS but are formed only when specific neurons are activated. Since acquired memories or "engrams" are also signaled in the form of S/P words, it follows that memories do not exist in $\mathrm{S} / \mathrm{P}$ word form as well.

Using the two cell model as the basic unit of cognition it has been suggested that the sites of formation of neuronal words are properties of stimulated neuronal receptors which upon activation initiate transmission of meaningful frequency-encoded messages. It has also been proposed that the site of acquired memories is different, and is associated with a normal word translation step that occurs in 
the dendrite-synapse-dendrite fields between individual neurons, where plasticity-related modifications in the structural and biochemical properties of these fields can result in an altered state containing memory engrams that are then stored in the altered fields.

As an example of memory storage and retrieval in the human CNS, in the absence of sound or light, it is possible to internally stimulate appropriate neurons and initiate the recall of a particular learned melody or a particular previously observed color by accessing meaningful memorized neuronal S/P word representations at $<1,000 \mathrm{~Hz}$ for the sounds and colors that exist at much higher electromagnetic spectrum frequencies. In these cases, although complex and involving many neurons in different brain areas, the memorized frequency-encoded S/P words for the appropriate information would be recalled upon afferent neuronal activations by being generated de novo as meaningful afferent $\mathrm{S} / \mathrm{P}$ words are passed through dendrite-synapse-dendrite memory-containing fields prior to being transmitted to the brain network for the required recall task. In summary, it is proposed that dendrite-synapse-dendrite fields between neurons are the major sites for storage of structural and biochemical modifications that can modulate afferent neuronal words and result in memory engrams. However, it follows that a memory itself is ephemeral and is reconstituted each time it is needed, neither residing in afferent neuronal S/P encoded words nor in dendrite-synapse-dendrite fields between neurons, but only existing as a momentary chimera consisting of both codes and fields, that can not be isolated by itself.

\section{Conclusions}

In this paper evidence has been provided that individual neurons possess language, and that the form of the language consists of encoded temporal electrophysiological action potentials or spikes (S) operating on a millisecond timescale that, along with pauses $(\mathrm{P})$ between spikes constitute a two letter "alphabet" that generates meaningful frequency-encoded signals or neuronal "words" in a primary S/P language for transmission to other neurons. The language characteristics of neurons appear to satisfy the biosemiotic requirement that individual cells can "produce meaningful signs" and transmit them to other cells that "can interpret the signs". It has also been observed that when a word from an afferent neuron is transmitted to an efferent neuron, it is usually translated into an equivalent frequency-encoded meaningful word, but in a different spike-pause language. Based on this observation, it is hypothesized that this inter-neuronal biosemiotic word translation step is important to brain function in that it allows for variations in meaning to occur. In this way, external sensor, or internal signaling experiences that induce changes in electrophysiological, structural or biochemical elements in the inter-neuronal signaling train can modify the meaning of an original word (learning), and lead to storage of the learned information of that experience (memory) in the form of stable long-term alterations in components of specific inter-neuronal dendritic-synaptic-dendritic fields.

While many investigations have focused on the whole brain with its billions of neuronal connections to try to understand biosemiotic interactions, in this article the focus is on the microscopic and sub-microscopic interfaces between individual neurons operating in a ms timeframe in order to explore how information is initially generated and then transmitted. The novel hypothesis offered in this paper is that individual neurons use language to communicate, and that there is a normal language translation step between neurons that plays an important role in communication as well as in learning and memory. Furthermore, that this inter-cellular process is also the basis for semiosis on the organ, organism, and 
inter-organism levels. Moreover, unlike whole brain studies of cognition involving many different disciplines, this hypothesis bridges the gap between measurable cognitive functioning and its underlying cellular processes. Lastly, this is a testable hypothesis that may be amenable to proof by mathematical modeling using relatively few variables [4,39], and on results of dynamic cell based inter-neuronal experimental data [26].

\section{Abbreviations}

$\begin{array}{ll}\text { AP's } & \text { action potentials; } \\ \text { AQP4 } & \text { aquaporin-4; } \\ \text { ATP } & \text { adenosine triphosphate; } \\ \text { CNS } & \text { central nervous system; } \\ \text { Glc } & \text { glucose }\end{array}$

\section{References}

1. Barbieri, M. The code model of semiosis: The first steps toward a scientific biosemiotics. Am. J. Semiotics 2008, 24, 1-3, 23-37.

2. Barbieri, M. Three types of semiosis. Biosemiotics 2009, 2, 19-30.

3. Averbeck, B.B.; Lee, D. Coding and transmission of information by neural ensembles. Trends Neurosci. 2004, 27, 225-230.

4. Nadasdy, Z. Information encoding and reconstruction from the phase of action potentials. Front. Syst. Neurosci. 2009, 3, doi:10.3389/neuro.06.006.2009.

5. Somogyi, P.; Klausberger, T. Defined types of cortical interneurone structure space and spike timing in the hippocampus. J. Physiol. (Lond) 2005, 562, 9-26.

6. Eyherabide, H.G.; Rokem, A.; Herz, A.V.M.; Samengo, I. Bursts generate a non-reducible spike pattern code. Front Neurosci. 2009, 3, 8-14, doi:10.3389/neuro.01.002.2009.

7. Clifford, C.W.; Ibbotson, M.R. Response variability and information transfer in directional neurons of the mammalian horizontal optokinetic system. Visual Neurosci. 2000, 17, 207-215.

8. Goldman, M.S.; Maldonado, P.; Abbott, L.F. Redundancy reduction and sustained firing with stochastic depressing synapses. J. Neurosci. 2002, 22, 584-591.

9. Lennie, P. The cost of cortical computation. Curr. Biol. 2003, 13, 493-497.

10. Borst, A. Noise, not stimulus entropy, determines neuronal information rate. J. Computational Neurosci. 2003, 14, 23-31.

11. Gillary, H.L. Stimulation of the salt receptor of the blowfly. II. Temperature. J. Gen. Physiol. 1966, 50, 351-357.

12. Laughlin, S.B.; Sejnowski, T.J. Communication in neuronal networks. Science 2003, 301, 1870-1874.

13. Ishimoto, H.; Tanimura, T. Molecular neurophysiology of taste in Drosophila. Cell. Mol. Life Sci. 2004, 61, 10-18.

14. Gilbertson, T.A.; Avenet, P.; Kinnamon, S.C.; Roper, S.D. Proton currents through amiloride-sensitive Na channels in hamster taste cells. J. Gen. Physiol. 1992, 100, 803-824. 
15. Merivee, E.; Renou, M.; Mand, M.; Luik, A.; Heidemaa, M.; Ploomi, A. Electrophysiological responses to salts from antennal chaetoid taste sensilla of the ground beetle Pterostichus aethiops. J. Insect Physiol. 2004, 50, 1001-1013.

16. Anand, B.K.; Chhina, G.S.; Sharma, K.N.; Dua, S.; Singh, B. Activity of single neurons in the hypothamic feeding centers: effect of glucose. Am. J. Physiol. 1964, 207, 1146-1154.

17. Levin, B.E.; Routh, V.H.; Kang, L.; Sanders, N.M.; Dunn-Meynell, A.A. Neuronal glucosensing, What do we know after 50 years? Diabetes 2004, 53, 2521-2528.

18. Ren, X.; Ligang, Z.; Terwilliger, R.; Newton, S.S.; de Araujo, I.E. Sweet taste signaling functions as a hypothalamic glucose sensor. Front. Integr. Neurosci. 2009, 3, doi:10.3389/ neuro.07.012.2009.

19. Baslow, M.H. A novel key-lock mechanism for inactivating amino acid neurotransmitters during transit across extracellular space. Amino Acids 2009. doi:10.1007/s00726-009-0232-0.

20. Baslow, M.H.; Guilfoyle, D.N. Using proton magnetic resonance imaging and spectroscopy to understand brain "activation". Brain Lang. 2007, 102, 153-164.

21. Di Lorenzo, P.M.; Leshchinskiy, S.; Moroney, D.N.; Ozdoba, J.M. Making time count: Functional evidence for temporal coding of taste sensation. Behav. Neurosci. 2009, 123, 14-25.

22. Wheatley, D.N. Diffusion theory, the cell and the synapse. BioSystems 1998, 45, 151-163.

23. Hormuzdi, S.G.; Filippov, M.A.; Mitropoulou, G.; Monyer, H.; Bruzzone, R. Electrical synapses: A dynamic signaling system that shapes the activity of neuronal networks. Biochim. Biophys. Acta 2004, 1662, 113-137.

24. Hausser, M.; Spruston, N.; Stuart, G.J. Diversity and dynamics of dendritic signaling. Science 2000, 290, 739-744.

25. Rumsey, C.C.; Abbott, L.F. Synaptic democracy in active dendrites. J. Neurophysiol. 2006, 96, 2307-2318.

26. Sjostrom, P.J.; Rancz, E.A.; Roth, A.; Hausser, M. Dendritic excitability and synaptic plasticity. Physiol. Rev. 2008, 88, 769-840.

27. Gidon, A.; Segev, I. Spike-timing-dependent synaptic plasticity and synaptic democracy in dendrites. J. Neurophysiol. 2009, 101, 3226-3234.

28. Komendantov, A.O.; Ascoli, G.A. Dendritic excitability and neuronal morphology as determinants of synaptic efficacy. J. Neurophysiol. 2009, 101, 1847-1866.

29. Baslow, M.H. Memory and enzyme induction. Science 1963, 139, 1091-1095.

30. Ly, C.V.; Verstreken, P. Mitochondria at the synapse. The Neuroscientist 2006, 12, 291-299.

31. Fellin, T. Communication between neurons and astrocytes: Relevance to the modulation of synaptic and network activity. J. Neurochem. 2009, 108, 533-544.

32. Ostby, I.; Oyehaug, L.; Einevoll, G.T.; Nagelhus, E.A.; Plahte, E.; Zeuthen, T.; Lloyd, C.M.; Ottersen, O.P.; Omholt, S.W. Astrocytic mechanisms explaining neural-activity-induced shrinkage of extraneuronal space. PLOS Comput. Biol. 2009, 5, 1-12.

33. Berry, M.J., II; Meister, M. Refractoriness and neural precision. J. Neurosci. 1998, 18, 2200-2211.

34. Zachariah, M.K.; Coleman, G.T.; Mahns, D.A.; Zhang, H.Q.; Rowe, M.J. Transmission security for single, hair follicle-related tactile afferent fibers and their target cuneate neurons in cat. $J$. Neurophysiol. 2001, 86, 900-911. 
35. Rosenzweig, M.R. Aspects of the search for neural mechanisms of memory. Ann. Rev. Psychol. 1996, 47, 1-32.

36. Kolb, B.; Whishaw, I.Q. Brain plasticity and behavior. Ann. Rev. Psychol. 1998, 49, 43-64.

37. Mora, F.; Gregorio, S.; del Arco, A. Aging, plasticity and environmental enrichment: Structural changes and neurotransmitter dynamics in several areas of the brain. Brain Res. Rev. 2007, 55, 78-88.

38. Baslow, M.H. NAAG peptidase as a therapeutic target: Potential for regulating the link between glucose metabolism and cognition. Drug News Perspect. 2006, 19, 145-150.

39. Kobayashi, R.; Tsubo, Y.; Shinomoto, S. Made-to-order spiking neuron model equipped with a multi-timescale adaptive threshold. Front. Comput. Neurosci. 2009, 3, doi:10.3389/neuro.10.009.2009.

(C) 2009 by the authors; licensee Molecular Diversity Preservation International, Basel, Switzerland. This article is an open-access article distributed under the terms and conditions of the Creative Commons Attribution license (http://creativecommons.org/licenses/by/3.0/). 